\title{
TECNOLOGIA DA INFORMAÇÃO E COMUNICAÇÃO E TECNOLOGIA ASSISTIVA: APROXIMAÇÕES E DISTANCIAMENTOS
}

\author{
TECNOLOGÍAS DE LA INFORMACIÓN Y LA COMUNICACIÓN Y LA TECNOLOGÍA \\ DE ASISTENCIA: APROXIMACIONES Y DISTANCIAS
}

\author{
INFORMATION AND COMMUNICATION TECHNOLOGY AND ASSISTIVE \\ TECHNOLOGY: CONVERGENCES AND DIVERGENCES
}

\author{
Thiago SARDENBERG ${ }^{1}$ \\ Helenice MAIA ${ }^{2}$
}

RESUMO: O artigo apresenta e discute aproximações e distanciamentos entre Tecnologia da Informação e Comunicação (TIC) e Tecnologia Assistiva (TA) no campo da Educação Especial na Perspectiva da Educação Inclusiva. Buscou-se evidenciar como estes termos foram empregados na Declaração Mundial de Educação para Todos, na Declaração de Salamanca, no Fórum Mundial de Educação de Dakar, na Convenção Internacional sobre os Direitos das Pessoas com Deficiência e seu Protocolo Facultativo, na Lei Brasileira de Inclusão da Pessoa com Deficiência e em distintas publicações da Organização das Nações Unidas para a Educação, a Ciência e a Cultura. Concluiu-se que há um complexo imbricamento entre essas duas modalidades de tecnologia, sendo ambos os termos amplamente empregados de maneira intrincada por profissionais com as mais distintas formações.

PALAVRAS-CHAVE: Tecnologia da Informação e Comunicação. Tecnologia assistiva. Marcos norteadores. Pessoa com deficiência.

RESUMEN: El artículo presenta y discute aproximaciones y distancias entre las Tecnologías de la Información y la Comunicación (TIC) y la Tecnología de Asistencia (TA) en el campo de la Educación Especial desde la Perspectiva de la Educación Integrada. Intentamos mostrar cómo estos términos fueron utilizados en la Declaración Mundial sobre Educación para Todos, en la Declaración de Salamanca, en el Foro Mundial de Educación en Dakar, en la Convención Internacional sobre los Derechos de las Personas con Discapacidad y su Protocolo Facultativo, en la Ley Brasileña para la Inclusión de Personas con Discapacidad y en diferentes publicaciones de las Organización de las Naciones Unidas para la Educación, la Ciencia y la Cultura. Se concluyó que existe una superposición compleja entre estas dos modalidades de tecnologías, y que ambos los términos se utilizan ampliamente de manera intrincada por profesionales con los más diferentes antecedentes.

\footnotetext{
${ }^{1}$ Instituto Benjamin Constant (IBC), Rio de Janeiro - RJ - Brasil. Professor de Orientação e Mobilidade lotado no Departamento de Estudos e Pesquisas Médicas e de Reabilitação. Doutorado em Educação (UNESA). ORCID: https://orcid.org/0000-0003-4341-4177. E-mail:tsardenberg@gmail.com

${ }^{2}$ Universidade Estácio de Sá (UNESA), Rio de Janeiro - RJ - Brasil. Professora do Programa de Pós-Graduação em Educação. Doutorado em Educação Escolar (UFRJ). ORCID: https://orcid.org/0000-0002-1169-9051. Email: helemaia@uol.com.br
}

RIAEE - Revista Ibero-Americana de Estudos em Educação, Araraquara, v. 16, n. esp. 4, p. 3072-3085, dez. 2021. e-ISSN: 1982-5587 
PALABRAS CLAVE: Tecnologías de la Información y la Comunicación. Tecnología de asistencia. Principios rectores. Personas con discapacidad.

ABSTRACT: This article presents and discusses convergences and divergences between Information and Communication Technology (ICT) and Assistive Technology (AT) in the field of Special Education from the perspective of Inclusive Education. We sought to show how these terms were used in the World Declaration on Education for All, in the Salamanca Statement, at the World Education Forum in Dakar, at the International Convention on the Rights of Persons with Disabilities (CRPD) and its Optional Protocol, in the Brazilian Law for the Inclusion of Persons with Disabilities and in different publications of the United Nations Educational, Scientific and Cultural Organization (UNESCO). It was concluded that there is a complex overlap between these two modalities of technology, and that both terms are widely used in an intricate manner by professionals with the most different backgrounds.

KEYWORDS: Information and Communication Technology. Assistive technology. Guiding milestones. Person with disabilities.

\section{Introdução}

Tecnologia de Informação e Comunicação (TIC) e Tecnologia Assistiva (TA) são tecnologias presentes em distintos espaços e amplamente empregadas, na atualidade, por diferentes profissionais. De forma restrita, TIC se refere aos meios técnicos que tratam a informação e promovem a comunicação, enquanto TA compreende tecnologias que visam à autonomia, independência, qualidade de vida e inclusão social de Pessoas com Deficiência (PcD). Estas pessoas são reconhecidas como aquelas que têm "impedimento de longo prazo de natureza física, mental, intelectual ou sensorial, o qual, em interação com uma ou mais barreiras, pode obstruir sua participação plena e efetiva na sociedade em igualdade de condições com as demais pessoas" (BRASIL, 2015).

A deficiência pode ser classificada a partir de dois modelos distintos. O modelo biomédico foi proposto na Classificação Internacional das Deficiências, Incapacidades e Desvantagens (CIDID, 1989), por meio de um modelo linear em que a deficiência está centrada na pessoa, em padrões de normalidade de funções psicológicas, fisiológicas ou anatômicas. O modelo biopsicossocial foi indicado na Classificação Internacional de Funcionalidade, Incapacidade e Saúde (CIF, 2003), tem como parâmetro a funcionalidade e a incapacidade associadas a uma condição de saúde, e a deficiência está centrada em fatores pessoais e ambientais que afetam ou podem afetar direta ou indiretamente as pessoas, a partir de barreiras atitudinais, tecnológicas, urbanísticas, arquitetônicas, nos transportes, nas comunicações e na informação, presentes nos mais diversos ambientes. 
As duas classificações foram propostas pela Organização Mundial de Saúde (OMS) e diferentes modalidades de tecnologias que promovam a inclusão das PcD estão previstas nos dois modelos, porém a maneira como elas foram intentadas é diferente. Em nossos estudos temos defendido o modelo biopsicossocial como o mais adequado para fomentar discussões a respeito da funcionalidade, da finalidade e da usabilidade dessas tecnologias.

Especificamente em relação à TIC e à TA, estas tecnologias apresentam-se como área de conhecimento interdisciplinar e não foram concebidas, exclusivamente, para a área educacional, mas fortemente incorporadas a ela, principalmente com o objetivo de favorecer o acesso à educação, facilitar o processo de ensino-aprendizagem e promover a equidade.

No intuito de verificar as possíveis aproximações e distanciamentos entre TIC e TA e como estas expressões foram empregadas em termos de sua funcionalidade e finalidade, foram analisados, em seu conteúdo (BARDIN, 2016), documentos essenciais para a orientação de ações voltadas para o uso destas tecnologias por $\mathrm{PcD}$, tanto em âmbito nacional quanto internacional. Consideramos que este estudo se justifica não apenas pela necessidade de abordar a relação entre TIC e TA em uma perspectiva conceitual, como pelo número reduzido de trabalhos que abordam a temática, sobretudo no campo da Educação Especial na Perspectiva da Educação Inclusiva, conforme apontam Oliveira e Mill (2016) e Nascimento e Nascimento (2018).

\section{Discutindo os conceitos de TIC e TA}

Informação e conhecimento tornaram-se imprescindíveis na contemporaneidade, assim como as TIC tornaram-se grandes provocadoras de mudanças sociais e dos modos de pensar e fazer educação.

A sigla TIC surgiu nos anos 1990 e foi utilizada, inicialmente, no Reino Unido, tendo sido posteriormente disseminada com a popularização da internet. Embora a Organização das Nações Unidas para a Educação, a Ciência e a Cultura (UNESCO, 2014) tenha previsto que qualquer definição estática desta modalidade tecnológica não auxiliaria muito a sua compreensão, as TIC podem ser definidas como

[...] todo e qualquer tipo de tecnologia que trate informação e auxilie na comunicação, podendo ser na forma de hardware, software, rede ou telemóveis, em geral. O termo TI, sem o $\mathrm{C}$ de comunicação, é ainda mais utilizado de forma geral e representa todos os recursos de tecnologia para $\mathrm{o}$ processamento de informações, incluindo softwares, hardwares, tecnologias de comunicação e serviços relacionados (SENAI, 2019).

RIAEE - Revista Ibero-Americana de Estudos em Educação, Araraquara, v. 16, n. esp. 4, p. 3072-3085, dez. 2021. e-ISSN: 1982-5587 
Entendidas como fundamentais para o desenvolvimento social e humano, as TIC tiveram sua origem nos anos 1970, com a criação dos microprocessadores e dos microcomputadores e, nesta mesma década, computadores foram utilizados para fins educativos.

Valente e Almeida (2020), ao discutirem a respeito de tecnologias da educação, revelam que, no Brasil, também nos anos 1970, o uso do computador teve início a partir de experiências em três universidades: Universidade Federal do Rio de Janeiro (UFRJ), Universidade Federal do Rio Grande do Sul (UFRGS) e Universidade Estadual de Campinas (UNICAMP), tendo como referência o que estava ocorrendo em outros países, como Estados Unidos da América (EUA) e França. Registraram que somente na década seguinte iniciativas direcionadas à inserção das TIC na educação básica foram efetivadas, a partir de políticas públicas do Ministério de Educação (MEC). Para estes autores, desde os anos de 1980 a educação é considerada um dos pilares das políticas de inclusão digital da população, por meio do fomento à investigação, formação profissional e programas de inserção de recursos tecnológicos, implantação de infraestrutura nas escolas, conexão à internet e formação de professores. Como já afirmava Bruzzi (2016, p. 476), “o impacto das TIC, na educação é, na verdade, um aspecto particular de um fenômeno muito mais amplo, relacionado com o papel dessas tecnologias na atual sociedade da informação".

Concernente à elaboração do conceito de TA no Brasil pelo Comitê de Ajudas Técnicas (CAT), foram utilizados três termos de referência: 'Ajudas Técnicas', 'Tecnologia Assistiva' e 'Tecnologia de Apoio'. Os membros do comitê constataram que esses termos são utilizados em países de língua inglesa, língua espanhola e língua portuguesa (Portugal), sendo encontrados, respectivamente, como Assistive Technology, Ayudas Técnicas e Tecnologia de Apoio nos seguintes documentos: Americans with Disabilities Act (ADA), Empowering Users Through Assistive Technology (EUSTAT) e Secretariado Nacional para a Reabilitação e Integração das Pessoas com Deficiência (SNRIPD) (BRASIL, 2009a).

O termo TA surgiu, inicialmente, na legislação americana em 1988, através da Public Law 100-407, que em conjunto com outras leis dos Estados Unidos da América (EUA) compõem a ADA. Neste documento, na Seção 3, intitulada Definições e Regras, a TA é caracterizada por duas palavras: dispositivo e serviço (AMERICAN WITH DISABILITIES $A C T, 2021)$.

O EUSTAT (2021), por sua vez, define a Tecnologia de Apoio enquanto produtos e serviços. O foco do estudo realizado foi o usuário dessa tecnologia no contexto educacional, bem como a produção de materiais educacionais para $\mathrm{PcD}$, seus familiares e cuidadores. 
Outro documento analisado pelo CAT foi o do SNRIPD. Essa Secretaria foi proposta pelo Decreto-Lei n. 35, de 2 de maio de 1996, no âmbito do Ministério da Solidariedade e Segurança Social de Portugal. Por esse mesmo Decreto foi criado o Conselho Nacional para a Reabilitação e Integração das Pessoas com Deficiência (CNRIPD), para definir e executar a política de reabilitação e integração das Pessoas com Deficiência. A definição de Ajudas Técnicas, na primeira publicação do MEC nesta área, é oriunda de Portugal e se refere a

[...] qualquer produto, instrumento, estratégia, serviço e prática, utilizado por Pessoas com Deficiência e pessoas idosas, especialmente produzidas ou geralmente disponíveis para prevenir, compensar, aliviar ou neutralizar uma deficiência, incapacidade ou desvantagem e melhorar a autonomia e a qualidade de vida dos indivíduos (BRASIL, 2009a, p. 15).

Note-se que os três conceitos abrangem questões distintas, porém complementares. A proposição da ADA se resume a dispositivos e serviços; a do EUSTAT, a produtos e serviços; e na do SPRIPD, o conceito é mais abrangente, englobando produto, instrumento, estratégia, serviço e prática. Entretanto, há uma diferença fundamental em relação à implementação do conceito de TA que deve ser considerada: enquanto nos EUA é uma legislação específica (ADA), que regula os direitos das PcD neste país, dentre eles o uso da TA, na Europa é um consórcio (EUSTAT), que amplia sua utilização por todo o continente, sob a forma de rede de apoio, denominada "Rede global de informação sobre produtos de apoio que promovam a autonomia de pessoas com incapacidade".

Tendo como parâmetro os três conceitos anteriores, o CAT propôs um conceito em uma perspectiva interdisciplinar, integrando saberes das áreas da Saúde, Educação, Engenharias, TIC, dentre outras, para efetivar o processo de inclusão da $\mathrm{PcD}$, qual seja:

[...] produtos, equipamentos, dispositivos, recursos, metodologias, estratégias, práticas e serviços que objetivem promover a funcionalidade, relacionada à atividade e à participação da pessoa com deficiência ou com mobilidade reduzida, visando à sua autonomia, independência, qualidade de vida e inclusão social (BRASIL, 2009a, p. 9).

O conceito de TA cunhado pelo CAT, bastante abrangente, assim como o de TIC, foi definido a partir da fusão dos três conceitos internacionais propostos pela ADA, pelo EUSTAT e pelo SNRIPD. Ocorre que ao propor esta definição, a realidade brasileira não foi considerada e certos elementos que compõem o conceito de TA possibilitam distintos entendimentos do que, efetivamente, se caracteriza como TA. Além disto, a estreita aproximação entre TA e TIC em alguns momentos, como o uso do computador e do celular, propicia apreensão equivocada a respeito da funcionalidade e finalidade da TA para as PcD. 


\section{O uso da TA e da TIC por Pessoas com Deficiência: marcos norteadores}

No relatório intitulado "Abrindo novos caminhos para o empoderamento: TIC no acesso à informação e ao conhecimento para as Pessoas com Deficiência", a UNESCO enfatiza a inclusão, mas também trata das políticas públicas e regulamentações para estas tecnologias, pois, quando utilizadas por $\mathrm{PcD}$, possibilitam a elas o acesso à informação e ao conhecimento, como indicado no título do documento. Esta Organização cujo objetivo precípuo é preservar os direitos humanos engenha as TIC como ferramentas capacitadoras que possibilitam a execução de práticas inovadoras e, no que concerne às $\mathrm{PcD}$, são consideradas TIC acessíveis aquelas concebidas a partir dos princípios do Desenho Universal (UNESCO, 2014). Aqui fica evidenciado um afastamento entre TA e TIC, pois ao conceber esta última a partir dos princípios do Desenho Universal, ambas são engendradas para qualquer pessoa, tenha deficiência ou não, diferente do que foi referenciado pelo CAT ao propor o conceito de TA definindo como seu público-alvo PcD ou pessoas com mobilidade reduzida.

No Brasil, inicialmente, o Desenho Universal foi compreendido como:

[...] concepção de espaços, artefatos e produtos que visam atender simultaneamente todas das pessoas com diferentes características antropométricas e sensoriais, de forma autônoma, segura e confortável, constituindo-se nos elementos ou soluções que compõem a acessibilidade (BRASIL, 2004).

Em 2015 este conceito foi revisado e incluído na Lei Brasileira de Inclusão da Pessoa com Deficiência (LBI) como “concepção de produtos, ambientes, programas e serviços a serem usados por todas as pessoas, sem necessidade de adaptação ou de projeto específico, incluindo os recursos de tecnologia assistiva" (BRASIL, 2015). Destaca-se nesta lei a ampliação do conceito e a inclusão dos recursos de TA no paradigma do Desenho Universal.

Ainda que o Desenho Universal não seja o cerne deste artigo, este conceito apresenta uma íntima relação com a TIC em sua usabilidade por PcD, além da própria TA, que deve ser compreendida na perspectiva deste paradigma, como apontado na LBI (BRASIL, 2015). A acessibilidade nas TIC prevê que todas as pessoas terão a possibilidade de acessar estas tecnologias e, a respeito dessas informações, a UNESCO declarou no relatório que

[...] a acessibilidade das TIC está relacionada com o conceito de Desenho Universal - o qual estabelece que o projeto e a criação de ambientes, produtos, aplicativos e conteúdo devem possibilitar a utilização por todas as pessoas, inclusive aquelas com deficiência, sem necessidade de modificações especiais. O paradigma do Desenho Universal nas TIC não estabelece a criação de um modelo que atenda a todos. Na verdade, ele está voltado a atender as necessidades de cada usuário, levando em conta $o$ 
desafio de que um conjunto de configurações pode ajudar um grupo de Pessoas com Deficiência, mas ser problemático para outro grupo (UNESCO, 2014, p. 76).

$\mathrm{Na}$ perspectiva de atendimento às especificidades do sujeito, o Desenho Universal apresenta um projeto flexível, permitindo que cada usuário se sinta contemplado nas suas preferências e habilidade individuais e, sobretudo, que tenha suas necessidades atendidas.

A UNESCO (2014) cita ainda que a TIC deve ser compreendida a partir de dois aspectos: na perspectiva inclusiva e na identificação de benefícios específicos no uso destas tecnologias para PcD. Essa perspectiva inclusiva é importante, pois transfere o foco da pergunta 'O que são as tecnologias?', pautada inicialmente na definição de um conceito, para outra, 'O que está sendo feito para tornar as tecnologias atuais e as que estão surgindo mais acessíveis, de baixo custo e relevantes?', enfocando a acessibilidade e a usabilidade por todos os sujeitos, $\mathrm{PcD}$ ou não. A primeira pergunta torna-se mais relevante para a avaliação, estabelecimento de parâmetros e o desenvolvimento de indicadores para os quais deve haver um acordo claro e harmonizado sobre o que será mensurado. A segunda evidencia preocupação com a personalização da tecnologia para atender a uma necessidade específica, de um público específico, sendo a TIC considerada TA. No relatório da UNESCO, o termo TA foi usado para hardware e software ou ferramentas usadas para o acesso aos dispositivos de informação e comunicação, o que deixa explícita a estreita relação entre elas que este documento pretendeu evidenciar. No tocante à Informática, ainda neste relatório, TIC e TA têm função sinergética, permitindo ao usuário o desempenho de uma função. Para tanto, é necessário acessibilizar os conteúdos para eliminar as barreiras tecnológicas, comunicacionais e informacionais presentes.

Embora a informação e a comunicação possam se efetivar por diferentes meios e de distintas maneiras, o relatório da UNESCO acabou por reduzir o conceito de TA à ferramenta, circunscrevendo-o ao campo da Informática. Porém, é preciso compreender a TA em sentido mais amplo, pois o acesso à informação e à comunicação é apenas uma das necessidades das PcDs. Especificamente em relação aos alunos com deficiência, a UNESCO (2011) recomendou que estes devem se 'auto adaptarem':

[...] por meio da identificação dos recursos computacionais que melhor se adequem às suas necessidades. A habilidade de personalizar a tecnologia para atender às suas preferências e necessidades pessoais é um aprendizado para a vida toda que beneficiará o aluno em sua jornada pelo sistema de ensino (UNESCO, 2011, p. 32).

Diversos marcos norteadores apoiam o uso das TIC na Educação de PcD, dentre eles, 
a Declaração Mundial de Educação para Todos (UNICEF,1990), aprovada pela Conferência Mundial sobre Educação para Todos, realizada em Jomtien, na Tailândia, em 1990. Esta declaração propôs um plano de ação para a satisfação das necessidades básicas de aprendizagem de crianças, jovens e adultos, e ressaltou a importância da formação/capacitação de profissionais da Educação para o seu uso, tanto na sala de aula quanto na gestão educacional, ampliando a possibilidade de utilização para além da relação professor e aluno. É possível identificar neste documento um afastamento entre TIC e TA, pois o uso das TIC foi proposto para todos, independentemente da condição de deficiência.

Outro marco é a Declaração de Salamanca, idealizada na Conferência Mundial de Educação Especial realizada em Salamanca, na Espanha, em 1994, com a participação de 88 governos, dentre eles o do Brasil, e 25 organizações internacionais, com a intenção de reafirmar o compromisso com a Educação para Todos, reconhecendo a necessidade e urgência do providenciamento de Educação para as crianças, jovens e adultos com necessidades educacionais especiais no sistema regular de ensino e reendossar a Estrutura de Ação em Educação Especial. Este documento indicou, dentre outras ações, o uso de tecnologia apropriada e viável que deveria ser usada, quando necessário, para aprimorar a taxa de sucesso escolar e auxiliar na comunicação, mobilidade e aprendizagem, além dos auxílios técnicos. No escopo da formação profissional foi salientada a importância do conhecimento e de habilidades requeridas para ensinar aos alunos com deficiência, incluindo a avaliação de necessidades especiais, a adaptação do conteúdo curricular, a utilização de tecnologia de assistência e a individualização de procedimentos de ensino no sentido de abarcar uma variedade maior de habilidades (ESPANHA, 1994).

Na Declaração de Salamanca há nítida divisão entre ‘tecnologia apropriada' utilizada em três grandes áreas do desempenho humano (comunicação, mobilidade e aprendizagem) e 'auxílios técnicos', embora o documento não explicite o que seria essa tecnologia ou estes auxílios. No entanto, a tecnologia de assistência citada no âmbito da formação de profissionais da Educação caracteriza o que atualmente denomina-se TA, pois refere-se à comunicação, mobilidade e aprendizagem do aluno com deficiência, enquanto os auxílios técnicos podem ser compreendidos como TIC, referindo-se a fatores relativos à escola. Aqui, identifica-se sinergia entre TIC e TA com o intuito de conceber a Educação Especial de uma nova maneira, conforme proposto nesta Declaração.

Decorrida uma década da Conferência Mundial de Educação para Todos, em que diversos países assumiram o compromisso de satisfazer as necessidades básicas de aprendizagem de crianças, jovens e adultos, erradicar o analfabetismo e universalizar o acesso 
à escolarização na infância, a UNESCO promoveu em todo o mundo amplo processo de avaliação dos progressos alcançados, que culminou no Fórum Mundial de Educação de Dakar, realizado em 2000 em Dakar, no Senegal. Neste Fórum, foi produzido um documento intitulado "Marco de Ação de Dakar", sendo este um compromisso coletivo assumido pelos países membros para alcançar os objetivos e metas da Educação para Todos. Uma das propostas foi adquirir novas TIC para apoiar o esforço em alcançar as metas firmadas (UNESCO, 2001). Porém, essas novas tecnologias não foram definidas, caracterizando a amplitude do conceito e possibilitando o uso de qualquer TIC na educação, independente das necessidades específicas de cada pessoa.

Em 2009, por meio do Decreto n. 6.949, o Brasil promulgou a Convenção Internacional sobre os Direitos das Pessoas com Deficiência e seu Protocolo Facultativo, assinados em Nova York, em 30 de março de 2007. Seu propósito é "promover, proteger e assegurar o exercício pleno e equitativo de todos os direitos humanos e liberdades fundamentais por todas as Pessoas com Deficiência e promover o respeito pela sua dignidade inerente" (BRASIL, 2009b). Nesta Convenção foram definidos cinco conceitos: comunicação, língua, discriminação por motivo de deficiência, adaptação razoável e desenho universal e, dentre eles, destacamos o primeiro:

\footnotetext{
"Comunicação" abrange as línguas, a visualização de textos, o braille, a comunicação tátil, os caracteres ampliados, os dispositivos de multimídia acessível, assim como a linguagem simples, escrita e oral, os sistemas auditivos e os meios de voz digitalizada e os modos, meios e formatos aumentativos e alternativos de comunicação, inclusive a tecnologia da informação e comunicação acessíveis (BRASIL, 2009b).
}

Como expresso no texto, há aproximação entre a TIC e a TA, pois "visualização de texto, o braille, a comunicação tátil, os caracteres ampliados, os dispositivos de multimídia acessível, os sistemas auditivos e os meios de voz digitalizada e os modos, meios e formatos aumentativos e alternativos de comunicação" caracterizam TA, enquanto a TIC acessível é a utilização destas tecnologias por PcD, configurando-se como TIC na perspectiva da TA ou TIC como TA.

Apesar dessa Convenção evidenciar que o acesso à TIC e à TA facilita o exercício de outros direitos, dentre eles o acesso à Educação, esses conceitos não foram definidos ou caracterizados. No artigo 4, Obrigações Gerais, constatam-se duas alíneas relacionadas ao uso de tecnologias: 
g) Realizar ou promover a pesquisa e o desenvolvimento, bem como a disponibilidade e o emprego de novas tecnologias, inclusive as tecnologias da informação e comunicação, ajudas técnicas para a locomoção, dispositivos e tecnologias assistivas, adequados a pessoa com deficiência, dando prioridade a tecnologias de custo acessível;

h) Propiciar informação acessível para as Pessoas com Deficiência a respeito de ajudas técnicas para locomoção, dispositivos e tecnologias assistivas, incluindo novas tecnologias bem como outras formas de assistência, serviços de apoio e instalações. (BRASIL, 2009b).

Porém, neste artigo, ajudas técnicas e dispositivos são citados, equivocadamente, apartados da TA. É necessário esclarecer que ajudas técnicas, assim como tecnologias de apoio, foram termos utilizados como sinônimos de TA até a definição do conceito pelo CAT em 2007, e dispositivo e serviço de apoio está englobado no conceito de TA, o que não justifica a utilização destes termos como tecnologias distintas.

Do mesmo modo foram previstos o uso de TIC para a promoção da acessibilidade e no tocante à mobilidade pessoal, "o acesso a tecnologias assistivas, dispositivos e ajudas técnicas de qualidade, e formas de assistência humana ou animal e de mediadores, inclusive tornandoos disponíveis a custo acessível" (BRASIL, 2009b). Aqui também ajudas técnicas e dispositivos aparecem apartados no conceito de TA, o que não procede, pois Ajudas Técnicas e TA são sinônimos e dispositivos é um dos elementos que compõem o conceito de TA.

Chama atenção em primeiro lugar o uso da assistência humana como TA, o que não aparece nos demais documentos consultados, e o animal, não necessariamente o cão-guia, conforme previsto no Decreto n. 5.904 (BRASIL, 2006). Em segundo lugar, o uso do termo tecnologia assistiva no plural contraria o proposto pelo CAT, que o cunhou no singular por compreendê-lo como uma área de conhecimento de característica interdisciplinar. Apesar de a Convenção sobre os Direitos das PcD ser internacional, não parece ter havido preocupação dos pesquisadores que elaboraram o documento em adequar a tradução ao conceito de TA cunhado no Brasil ou de oferecer algum tipo de esclarecimento por meio de notas do tradutor, informando que esse conceito difere entre países.

Mais recentemente, em 2015, a LBI também previu o uso da TA, da TIC e da Tecnologia Social (TS), embora essas duas últimas não fossem definidas ou caracterizadas nesta lei. Em relação à TA, há um capítulo específico versando sobre ela, o capítulo III, intitulado 'Da Tecnologia Assistiva'. A expressão tecnologia assistiva aparece no texto ora no singular, ora no plural, e na maior parte das vezes o conceito se reduz a recurso. Com relação às TIC, estas são empregadas como instrumentos de superação de limitações funcionais e de barreiras à comunicação, à informação, à Educação e ao entretenimento da $\mathrm{PcD}$. 
Diferentemente dos outros documentos analisados, a LBI aparta TIC e TA enquanto tecnologias independentes (BRASIL, 2015).

Após a análise destes marcos norteadores, foi possível evidenciar distintas aproximações e distanciamentos entre TIC e TA, o que provoca um enredamento e, por vezes, um imbricamento entre essas duas tecnologias, tornando confusas questões relativas à funcionalidade, finalidade e usabilidade dessas modalidades de tecnologias, sobretudo pelas PcD.

\section{Considerações finais}

Conforme anunciado ao longo do texto, TIC e TA são termos amplamente empregados de maneira indistinta, como é o caso do uso do computador e do celular pelas PcD. Dependendo do tipo de deficiência e da maneira como o usuário utiliza o computador e o celular, estes artefatos podem ou não se configurar como TA. Para que possa ser caracterizada como TA e acima de tudo utilizada de maneira adequada, é necessário que se tenha em conta quem é o usuário, a funcionalidade da tecnologia, o contexto em que ela está sendo empregada e qual a finalidade desse emprego.

TIC podem ser utilizadas por qualquer usuário. Embora os documentos consultados apresentem o público-alvo destas tecnologias, não há, em vários momentos, distinção do que seja uma ou outra, além do uso indiscriminado da TIC na perspectiva da TA ou como TA, o que possibilita o emprego destes termos de maneira emaranhada por profissionais com as mais distintas formações.

Para Sardenberg e Maia (2019, p. 7),

[...] pensar em tecnologia(s) assistiva(s) é refletir sobre o que elas podem "fazer" ou "trazer" para todos nós, sujeitos [considerados] com deficiência ou não. É entender que o computador, o celular, os programas e a internet, por si só, não configuram tecnologias assistivas. Talvez possamos identificálos como ferramentas potentes para nos "colocar" e "viver" no mundo.

Embora o conceito de TA esteja especificamente relacionado às $\mathrm{PcD}$ ou com mobilidade reduzida, como proposto pelo CAT em 2007, estes autores defendem que TA, tomando como base o modelo biopsicossocial da deficiência, engloba diferentes perspectivas de saúde (biológica, individual e social), uma vez que o objetivo fundante deste conceito é a promoção da autonomia, independência, qualidade de vida e inclusão social.

Pessoas que não são consideradas deficientes à luz do modelo biomédico, como aquelas que apresentam transtornos de aprendizagem, por exemplo, são consideradas 
deficientes na proposta do modelo biopsicossocial. Além disso, de acordo com LBI (BRASIL, 2015), essas pessoas têm impedimentos de longo prazo de naturezas distintas, que em interação com uma ou mais barreiras podem ver-se parcial ou totalmente impedidas de participar da sociedade em igualdade de condições com as demais. Portanto, pessoas com dislexia, discalculia, dentre outros transtornos, podem se beneficiar da TA, especialmente nos contextos escolares, uma vez que esta tecnologia pode possibilitar e ampliar sua participação efetiva no processo educacional.

\section{REFERÊNCIAS}

AMERICAN WITH DISABILITIES ACT. Disponível em:

https://www.ada.gov/2010_regs.htm. Acesso em: 12 maio 2021.

BARDIN, L. Análise de Conteúdo. São Paulo: Edições 70, 2016.

BRASIL. Presidência da República. Decreto n. 5.296, de 2 de dezembro de 2004.

Regulamenta as Leis $\mathrm{n}^{\mathrm{o}} \mathrm{s} 10.048$, de 8 de novembro de 2000 , que dá prioridade de atendimento às pessoas que especifica, e 10.098, de 19 de dezembro de 2000, que estabelece normas gerais e critérios básicos para a promoção da acessibilidade das pessoas portadoras de deficiência ou com mobilidade reduzida, e dá outras providências. Brasília, DF: Presidência da República, 2004. Disponível em: www.planalto.gov.br/ccivil_03/_ato20042006/2004/decreto/d5296.htm. Acesso em: 20 maio 2019.

BRASIL. Presidência da República. Casa Civil. Decreto n.5.904, de 21 de setembro de 2006. Regulamenta a Lei $n^{\circ} 11.126$, de 27 de junho de 2005, que dispõe sobre o direito da pessoa com deficiência visual de ingressar e permanecer em ambientes de uso coletivo acompanhada de cão-guia e dá outras providências. Brasília, DF: Presidência da República/ Casa Civil, 2006. Disponível em: planalto.gov.br/ccivil 03/ ato2004-

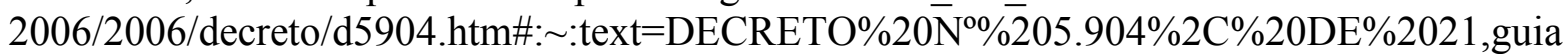
\%20e\%20dá\%20outras\%20providências. Acesso em: 21 nov. 2020.

BRASIL. Presidência da República. Casa Civil. Decreto n. 6.949, de 25 de agosto de 2009. Promulga a Convenção Internacional sobre os Direitos das Pessoas com Deficiência e seu Protocolo Facultativo, assinados em Nova York, em 30 de março de 2007. Brasília, DF: Presidência da República/Casa Civil, 2009b. Disponível em: http://www.planalto.gov.br/ccivil_03/_ato2007-2010/2009/decreto/d6949.htm. Acesso em: 03 abr. 2021.

BRASIL. Presidência da República. Lei n. 13.146, de 6 de julho de 2015. Institui a Lei Brasileira de Inclusão da Pessoa com Deficiência (Estatuto da Pessoa com Deficiência). Brasília, DF: Presidência da República, 2015. Disponível em: www.planalto.gov.br/ccivil_03/_Ato2015-2018/2015/Lei/L13146.htm. Acesso em: 10 maio 2019.

BRASIL. Subsecretaria Nacional de Promoção dos Direitos da Pessoa com Deficiência. Comitê de Ajudas Técnicas. Tecnologia Assistiva. Brasília: CORDE, 2009a. 138 p. 
BRUZZI, D. G. Uso da tecnologia na educação, da história à realidade atual. Polyphonía, v. 27, n. 1, p. 475-483, jan./ jun. 2016. Disponível em:

https://www.revistas.ufg.br/sv/article/view/42325. Acesso em: 10 maio 2021.

CIDID. Classificação internacional das deficiências, incapacidades e desvantagens

(HANDICAPS): um manual de classificação das consequências das doenças. Lisboa: Organização Mundial de Saúde, 1989.

CIF. Classificação Internacional de Funcionalidade. Incapacidade e saúde. São Paulo: Editora da Universidade de São Paulo, 2003.

EMPOWERING users throug hassistive technology. Disponível em:

http://www.siva.it/research/eustat/portugue.html. Acesso em: 12 maio 2021.

ESPANHA. Declaração de Salamanca. Sobre Princípios, Políticas e Práticas na Área das Necessidades Educativas Especiais. Espanha: Salamanca, 1994. Disponível em: http://portal.mec.gov.br/seesp/arquivos/pdf/salamanca.pdf. Acesso em: 13 abr. 2021.

NASCIMENTO, R. A. L.; NASCIMENTO, G. V. S. Tecnologia Assistiva no Brasil (1999 a 2016): Mapeamento das produções e indicativos para novas pesquisas. In: CONGRESSO BRASILEIRO DE EDUCAÇÃO ESPECIAL, 8., 2018, São Carlos. Anais [...]. Campinas, SP: Galoá, 2018. Disponível em: https://proceedings.science/cbee/cbee2018/papers/tecnologia-assistiva-no-brasil--1999-a-2016---mapeamento-das-producoes-eindicativos-para-novas-pesquisa. Acesso em: 19 nov. 2021.

OLIVEIRA, C. D.; MILL, D. Acessibilidade, inclusão e tecnologia assistiva: um estudo bibliométrico. Revista Ibero-Americana de Estudos em Educação, Araraquara, v. 11, n. 3, p. 1169-1183, 2016. Disponível em:

https://periodicos.fclar.unesp.br/iberoamericana/article/view/8194. Acesso em: 10 nov. 2021.

SARDENBERG, T.; MAIA, H. Uma porta aberta: representações sociais de Tecnologia Assistiva. 1. ed. Curitiba, PR: Appris, 2019.

SENAI. Serviço Nacional de Aprendizagem Industrial. Mas afinal de contas, o que é TIC? Instituto SENAI de Inovação para Tecnologias da Informação e Comunicação. SENAI, 2019. Disponível em: https://isiTIC.com/2019/07/01/mas-afinal-de-contas-o-que-e-TIC/. Acesso em 26 mar. 2021.

UNESCO. Organização das Nações Unidas para a Educação, a Ciência e a Cultura. Educação para Todos: o compromisso de Dakar. Brasília, DF: UNESCO, 2001. Disponível em:

https://www.mprj.mp.br/documents/20184/1330730/2000_declaracaosobreeducacaoparatodos ocompromissodedakar.pdf. Acesso em: 01 abr. 2021.

UNESCO. Organização das Nações Unidas para a Educação, a Ciência e a Cultura. TIC Acessíveis e Ensino Personalizado para Alunos com Deficiências: um diálogo entre Educadores, Indústria, Governo e Sociedade Civil. Relatório da Reunião de Consultoria Especializada. Paris, 2011. Disponível em:

http://www.unesco.org/new/fileadmin/MULTIMEDIA/HQ/CI/CI/pdf/accessible_ict_students _disabilities_pt.pdf. Acesso em: 27 mar. 2021. 
UNESCO. Organização das Nações Unidas para a Educação, a Ciência e a Cultura. Abrindo novos caminhos para o empoderamento: TIC no acesso à informação e ao conhecimento para as Pessoas com Defiência. Relatório Global UNESCO. Comitê Gestor da Internet no Brasil. São Paulo, 2014. Disponível em:

https://cetic.br/es/media/docs/publicacoes/8/Relatorio_Global_Unesco_FINAL.pdf. Acesso em 21 mar. 2021.

UNICEF. Fundo das Nações Unidas para a Infância. Declaração Mundial sobre Educação para todos. Conferência de Jomtien, 1990. Disponível em:

https://www.unicef.org/brazil/declaracao-mundial-sobre-educacao-para-todos-conferencia-dejomtien-1990. Acesso em: 01 abr. 2021.

VALENTE, J. A.; ALMEIDA, M. E. B. Políticas de tecnologia na educação no Brasil: visão histórica e lições aprendidas. Arquivos Analíticos de Políticas Educativas, Arizona State University, v. 28, n. 94, jun. 2020. Disponível em:

https://epaa.asu.edu/ojs/article/view/4295/2460. Acesso em: 29 mar. 2021.

\section{Como referenciar este artigo}

SARDENBERG, T.; MAIA, H. Tecnologia da informação e comunicação e tecnologia assistiva: aproximações e distanciamentos. Revista Ibero-Americana de Estudos em Educação, Araraquara, v. 16, n. esp. 4, p. 3072-3085, dez. 2021. e-ISSN: 1982-5587. DOI: https://doi.org/10.21723/riaee.v16iesp.4.16068

Submetido em: 15/09/2021

Revisões requeridas em: 30/10/2021

Aprovado em: 10/12/2021

Publicado em: 30/12/2021 